\title{
ARTICLE
}

\section{Reliability and use of INCL4.6-Abla07 spallation model in the frame of European Spallation Source target design}

\author{
Anne Leprince $^{\mathrm{a}^{*}}$, Jean-Christophe David ${ }^{\mathrm{a}}$, Daniel Ene ${ }^{\mathrm{b}}$ and Sylvie Leray ${ }^{\mathrm{a}}$ \\ ${ }^{a}$ Commissariat à l'Energie Atomique, CEA/Saclay, IRFU/Service de Physique Nucléaire, F-91191 Gif-sur-Yvette Cedex, \\ France; ${ }^{b}$ European Spallation Source ESS AB, P.O Box 176, SE-221 00 Lund, Sweden
}

\begin{abstract}
In the framework of the European Spallation Source (ESS) project, works on ESS target design need computational tools and the validation of these tools must be done to estimate accuracy of the calculation results. INCL4.6 and Abla07 have been implemented in a beta version of MCNPX2.7 to be able to use them when transport is needed as ESS spallation target design. Within ESS project, we decided to benchmark our spallation model on excitation functions $(\mathrm{p}+\mathrm{W})$ to assess the reliability on a thin target according to the produced isotope and the projectile energy. We focused on a list of hazardous nuclei given by previous ESS calculations and from previous experiments. These results, which can of course help to estimate uncertainties as designing ESS target, were supplemented by another benchmark done with a thick tungsten target and giving isotope production along the beam axis and radially as well.
\end{abstract}

\section{Keywords: spallation model; INCL4.6; Abla07; MCNPX; ESS; tungsten target; design}

\section{Introduction}

The European Spallation Source (ESS) is planned to be build and operated by the end of this decade at Lund in Sweden and will be the brightest source of neutrons in the world. This goal can only be achieved by designing this facility with the most reliable tools available. As regards the spallation target the reaction models must provide not only the right neutrons flux and spectra, but also well estimate the isotope production yields. This last point is important from the safety point of view (radiation, target radioactivity, gas and volatile production, etc.).

The spallation model combination INCL4.2-Abla [1-2] was known as a good spallation model and was implemented in the transport code MCNPX2.5.0 [3] (and following versions). Since then both parts have been still improved and the results showed that this new combination, INCL4.5-Abla07, was one of the most reliable spallation models during the IAEA Benchmark of spallation models [4]. The main difference with the previous versions was much better production of light and intermediate mass fragments $(\mathrm{A}<20)$. Finally the new INCL4.6 version is able also to treat well the low energy composite particle $\left(\mathrm{d}, \mathrm{t},{ }^{3} \mathrm{He}\right.$, alpha) as projectile. These last particles are produced by the primary beam interaction and could be responsible of specific isotope production.

As regards ESS target simulations, INCL4.6-Abla07

*Corresponding author. Email: anne.leprince@cea.fr
[5-6] was then interesting to use it after its implementation in a transport code. This has been done in a beta version of MCNPX2.7 [3]. The first point was to check the confidence level of INCL4.6-Abla07 on the isotope production in the reaction $\mathrm{p}+\mathrm{W}$, tungsten being the ESS target material, with focus on gaseous elements and hazardous. The second was to use this model combination for the calculation of the ESS target.

\section{Benchmarking of the new INCL4.6-Abla07}

To begin this part, it is worthwhile to present the ESS previous list of radionuclides production of gaseous elements (Table 1) and of hazard radionuclides into the ${ }^{\text {nat }} \mathrm{W}$ target (Table 2) [7]. These hazardous isotopes are ranked in the most numerous and dangerous (contribution of the radionuclide to total hazard index). Regarding isotope on light blue background model INCL4.6-Abla07 cannot, in its current version, estimate the production cross sections of a metastable state.

Table 1. Previous compilation of gaseous elements taken into account in our study of benchmarking on ${ }^{\text {nat }} \mathrm{W}$.

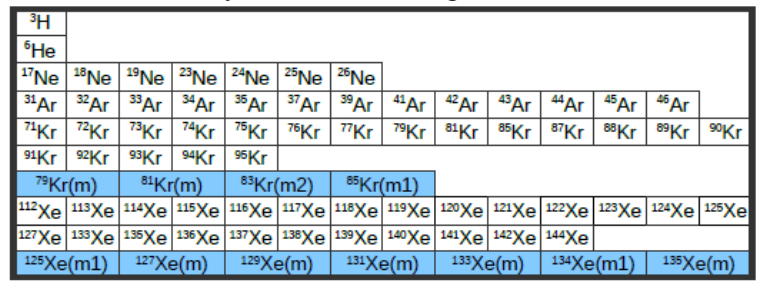


Table 2. Previous compilation of hazardous dominating isotopes taken into account in our study of benchmarking on

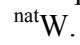

\begin{tabular}{|c|c|c|c|c|c|c|c|c|c|c|c|}
\hline${ }^{187} \mathrm{~W}$ & ${ }^{148} \mathrm{Gd}$ & ${ }^{186} \mathrm{Re}$ & ${ }^{182} \mathrm{Ta}$ & ${ }^{172} \mathrm{Hf}$ & ${ }^{185} \mathrm{~W}$ & ${ }^{188} \mathrm{Re}$ & \multicolumn{2}{|c|}{${ }^{178} \mathrm{Hf}(\mathrm{ml})$} & \multicolumn{2}{|c|}{${ }^{179} \mathrm{Hf}(\mathrm{ml})$} & ${ }^{169} \mathrm{Yb}$ \\
\hline $17,63 \%$ & $14,14 \%$ & $12,04 \%$ & $7,85 \%$ & $7,79 \%$ & $5,97 \%$ & $5,16 \%$ & 4,6 & & 2.6 & & 2,2 \\
\hline${ }^{183} \mathrm{Ta}$ & 1755 $\mathrm{Hf}$ & ${ }^{173} \mathrm{Lu}$ & 125 & ${ }^{179} \mathrm{Ta}$ & ${ }^{146} \mathrm{Gd}$ & ${ }_{172} \mathrm{Lu}$ & ${ }^{171} \mathrm{Lu}$ & ${ }^{149} \mathrm{~Tb}$ & ${ }^{170} \mathrm{Tm}$ & ${ }^{170} \mathrm{Lu}$ & 167T \\
\hline $2,21 \%$ & $1,61 \%$ & $1,47 \%$ & $1,32 \%$ & $1.06 \%$ & $0.82 \%$ & $0,72 \%$ & $0.62 \%$ & $0.50 \%$ & $0,44 \%$ & $0.42 \%$ & 0,3 \\
\hline
\end{tabular}

\subsection{On thin tungsten target}

Excitation functions, one of the basic data sets for modeling the activation yields, correspond to production cross sections for a given isotope and a given target according to energy projectile. There are two types of production cross section, independent and cumulative. The latter corresponds to the sum of the independent yields for a nucleus and all of its radioactive parents. Using the cascade model INCL4.6 and the deexcitation code Abla07 combination, the cross sections were calculated, for the production of nuclei in ${ }^{\text {nat }} \mathrm{W}$ by 30 $\mathrm{MeV}$ to $2.8 \mathrm{GeV}$ proton induced reactions. Then results obtained by the theoretical simulations were compared with the experimental data available in the literature. Two sets of data of ${ }^{\text {nat }} \mathrm{W}$ irradiated with few tens $\mathrm{MeV}$ to $2.6 \mathrm{GeV}$ protons were found in references [8] and [9].

As example Figure 1 shows the ratio between ${ }^{183} \mathrm{Ta}$ production cross-sections in natural tungsten calculated with INCL4.6-Abla07 compared to the experimental data [8-9]. Three lines were added to emphasize ratios equal to $2(0.5)$ and 1 . The ratios obtained are next to 2 between 100 and $2600 \mathrm{MeV}$ but increase when going under $100 \mathrm{MeV}$. In these cases simulation overestimates the measured results.

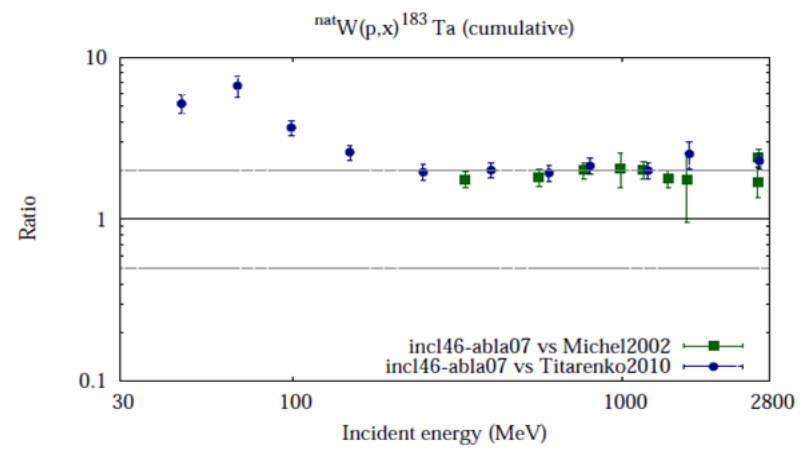

Figure 1. Ratios between ${ }^{183} \mathrm{Ta}$ production cross-section in ${ }^{\text {nat }} \mathrm{W}$ calculated with the new version INCL4.6-Abla07 and experimental data [8-9].

Unfortunately, there is no experimental data for the gaseous nuclides (Table 1), five isotopes of xenon excepted. Thereafter some results on ${ }^{\text {nat }} \mathrm{Pb}$ by $35 \mathrm{MeV}$ to $3 \mathrm{GeV}$ protons induced reactions will be presented. They give hints on the quality of the simulation for these gaseous radionuclides. The results showed hereafter (Figure 2) present the ratio between model results and experimental data for tritium [10]. The agreement is very good all along the energy range as evidenced by the ratio values close to 1 .

Finally on all residue production yields using a tungsten or lead target we can conclude that our excitation functions are in good agreement with experimental data available. Furthermore the average ratio is qualitatively of the order of 2.

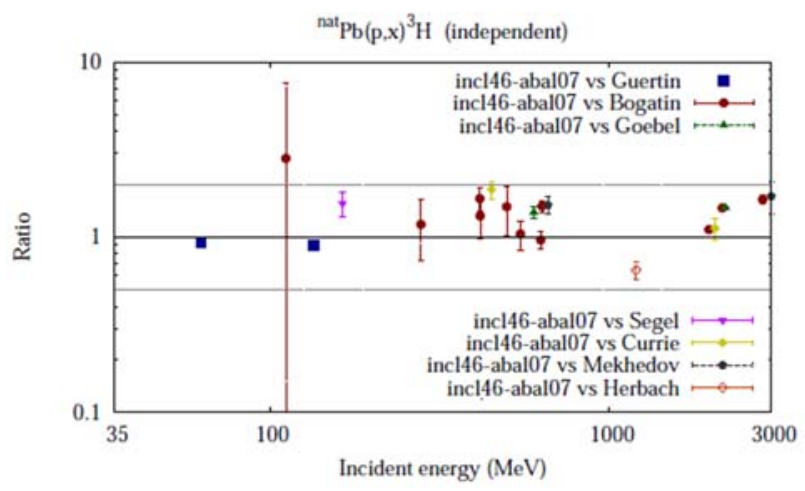

Figure 2. Ratio between tritium production cross-section in ${ }^{n a t} \mathrm{~Pb}$ calculated with the new version INCL4.6-Abla07 and experimental data measured by different groups [10].

\subsection{On thick tungsten target}

We have seen previously than INCL4.6-Abla07 reproduced very well the different data found in the literature. But this analysis is not totally complete because it doesn't take into account the reaction produced by other particles than protons. However it stays a good evaluation and both are complementary. In addition, only one experiment used a thick tungsten target [11] and experimental data are not available for all hazardous contaminant nuclei and gaseous elements of the ESS list (Table 1-2). We also consider an other experiment with thick $\mathrm{Hg}$ target with $\mathrm{Pb}$ and $\mathrm{Bi}$ samples irradiated by $2.83 \mathrm{GeV}$ protons [12] to draw useful information on the reliability of the INCL4.6-Abla07 model combination implemented in MCNPX, because it is a major issue to determine the uncertainty while comparing with the model. The study on this second set of data is in progress, so only preliminary results for thick tungsten target experiment will be discussed.

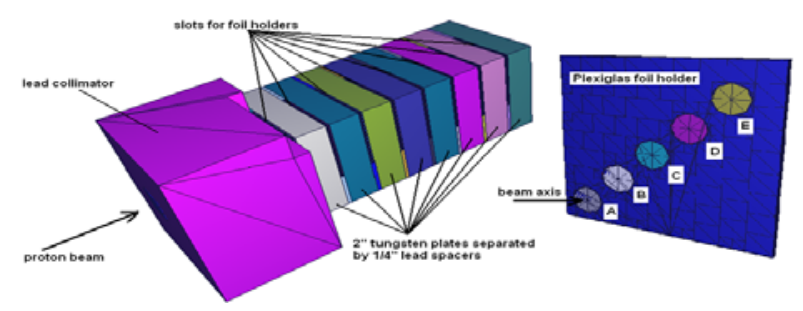

Figure 3. Drawing of the tungsten target and of tungsten target holder used in the experiment conducted by J.L. Ulmann et al. [11].

The experiment was done using proton beam delivered by the linear accelerator at the WNR (Weapons Neutron Research) facility at LAMPF [11]. Thick natural tungsten target were irradiated with $800 \mathrm{MeV}$ protons (Figure 3). Thin foils were inserted at various locations to sample the radiation environment inside the target. The foils inserted on the beam axis were to measure interactions due 
primarily to the incident beam; the off-axis foils sampled interactions due to scattered and secondary particles (primarily neutrons.) The tungsten foils were located 5.1, $10.2,15.2$, and $20.3 \mathrm{~cm}$ into the stack and centered on the beam axis and at radii of 2.14 (A), 4.28 (B), 6.43, and $8.57 \mathrm{~cm}$ from the axis.
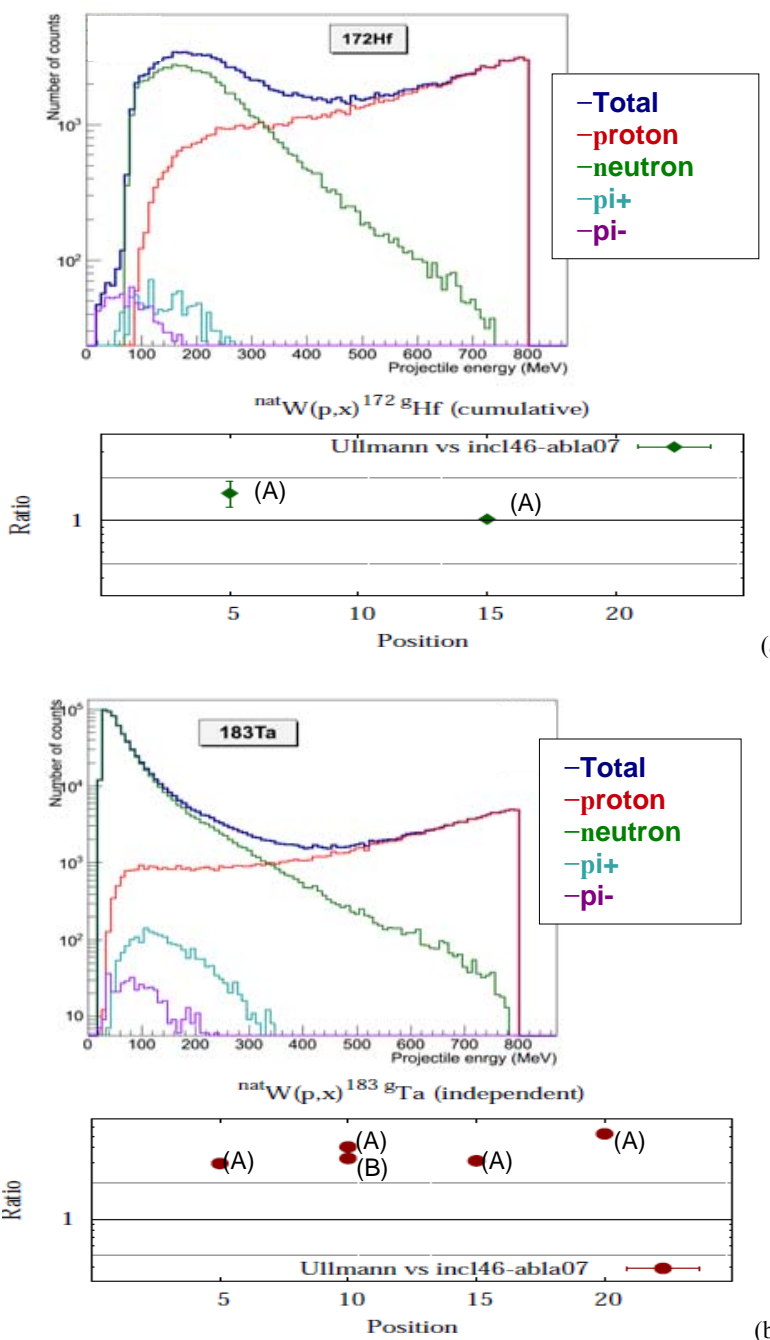

(b)

Figure 4. On top: respective and total numbers of reactions due to the interactions of the different particles involved in a thick W target bombarded by a $800 \mathrm{MeV}$ beam for ${ }^{172} \mathrm{Hf}$ (a) and ${ }^{183} \mathrm{Ta}$ (b) production. On bottom: ratio between ${ }^{172} \mathrm{Hf}$ cumulative (respectively ${ }^{183} \mathrm{Ta}$ independent) spallation yield (a) (respectively (b)) in ${ }^{\text {nat }} \mathrm{W}$.

Results presented in this part are obtained with INCL4.6-Abla07-MCNPX. This calculation takes into account the interaction of proton, neutron higher than 25 $\mathrm{MeV}$ and pion. We need to use CINDER'90 to consider low energy neutron, in this goal we are in relation with J.L. Ulmann et al. [11] to obtain irradiation and shut-down time used in this experiment. So results present here after should be refined and are a little bite lower than expected. Figure 4a shows the ratio between ${ }^{172} \mathrm{Hf}$ independent spallation yields in ${ }^{\text {nat }} \mathrm{W}$ calculated with INCL4.6-Abla07-MCNPX compared to the experimental data [11]. The agreement is very good and the ratio is below than 2 . The ${ }^{172} \mathrm{Hf}$ is mainly produced by high energy protons and neutrons above $100 \mathrm{MeV}$. In the case of ${ }^{183} \mathrm{Ta}$ (Figure $4 \mathbf{b}$ ), the production of this isotope is principally due to low energy neutrons (below $100 \mathrm{MeV}$ ) and we can notice that the ratio is higher than 2 , we have the right order of magnitude but we are less good as expected (Figure 1). We can also notice that in this case we overestimate the data, it is not a real problem as we consider the safety point of view. Furthermore we can notice the correspondence with excitation function (Figure 1) where ratios increase when proton energy is lower than, $100 \mathrm{MeV}$. It seems that INCL4.6-Abla07 gives less good estimations for low projectile.

In conclusion, even if these results are preliminary, the agreement between experimental data and the model combination INCL4.6-Abla07 in MCNPX is quite good. However we need to be more careful with nuclei produced mainly by low energy projectile.

\section{ESS calculations}

To explore the general features of radionuclide production, we performed INCL4.6-Abla07-MCNPX calculations in the detailed ESS geometry (Figure 5) and then used CINDER'90. The target is composed by 11 tungsten layers surrounded by $2 \mathrm{~mm}$ of helium. The first to fifth layers are $1.5 \mathrm{~cm}$ thick, the sixth and seventh $2 \mathrm{~cm}$, the eighth $3 \mathrm{~cm}$, the ninth $4 \mathrm{~cm}$, the tenth $6 \mathrm{~cm}$ and finally the eleventh $10 \mathrm{~cm}$.

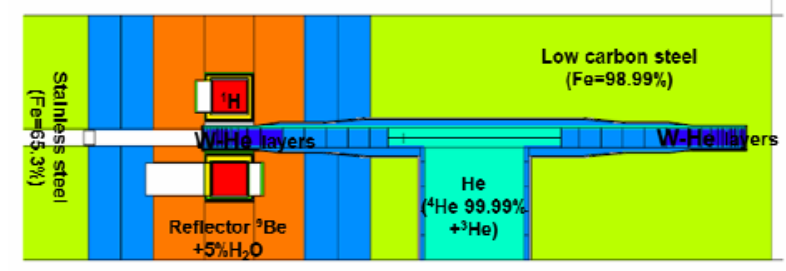

Figure 5. Drawing of MCNPX geometry of ESS tungsten target in $\mathrm{YZ}$ view.

These calculations demonstrate the properties of spallation with $2.5 \mathrm{GeV}$ protons, and subsequent transport and interactions of the secondary particles. In this way, we assess activity, production cross section of spallation products (beam on and beam off) and then we can validate the existing contaminant list [7] or define a new one, by calculation the hazard index (Eq. 1). The hazard index (HI) is defined as the sum over all the radionuclides present of the net capacity to conduct to radiological dose through inhalation:

$$
H I=\sum\left(A_{i} \times R F_{i} \times D C F_{i}\right)
$$

where: $A_{i}$ is the activity $(B q)$ of the radionuclide $i$ present in the target at the moment of evaluation, $R_{i}$ is the release fraction for the radionuclide $\mathrm{i}$ for a given accident or for representative accident; $\mathrm{DCF}_{\mathrm{i}}$ is the effective dose commitment per inhaled activation for the radionuclide $\mathrm{i}(\mathrm{Sv} / \mathrm{Bq})$. The product defined above is summed over all radionuclide present in the target at the moment of evaluation. To be on the safe side, conservative value were considered by taken as reference time for evaluations the end of the life of the target. The calculation of hazard index is in progress and 
will not be presented here.
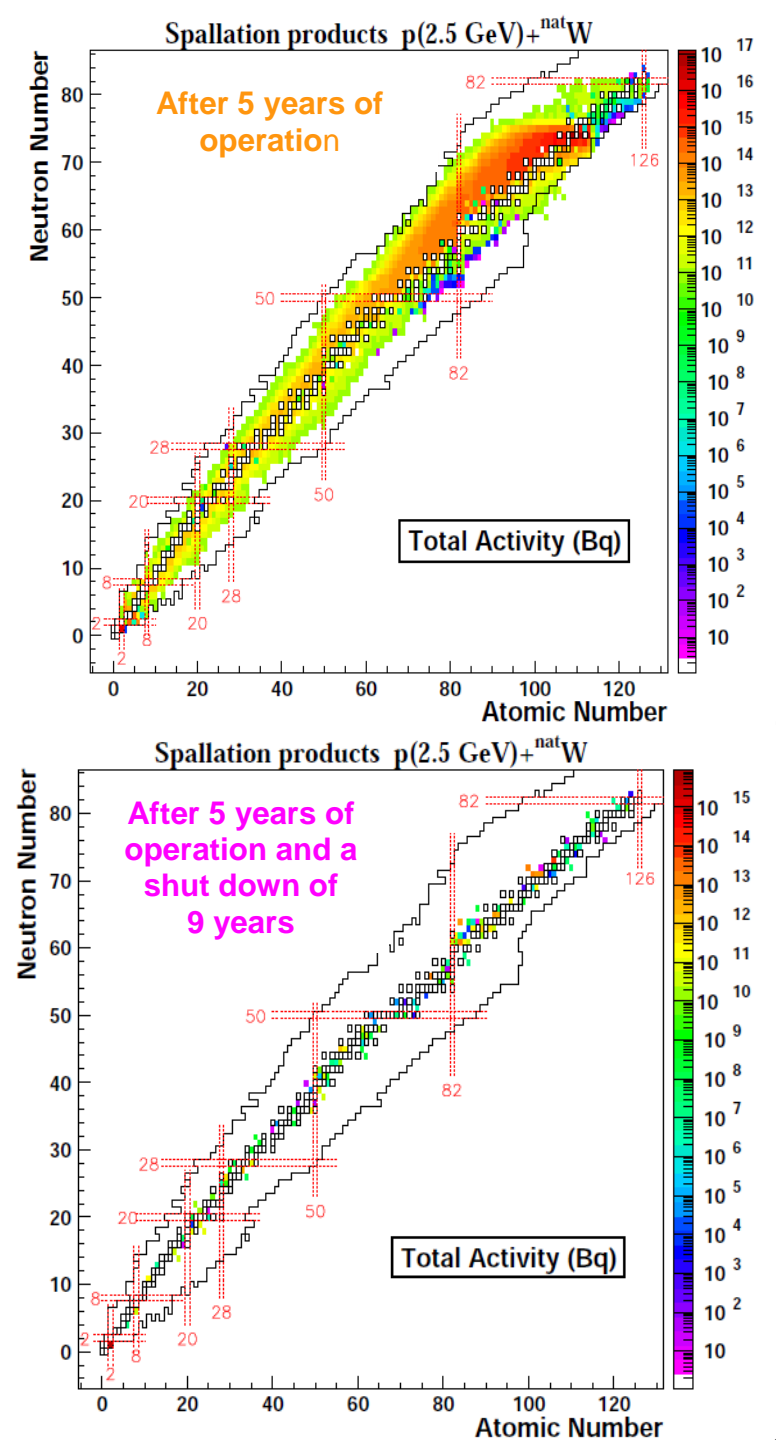

(b)

Figure 6. Nuclide total activity (Bq) of ESS tungsten target after 5 years of operation (a), and after 5 years of operation and a shut-down of 9 years (b), obtained by INCL4.6-Abla07 in MCNPX+CINDER'90 on a chart of nuclides.

The activity of spallation products in ESS tungsten target are represented on a nuclear-chart, the empty squares indicating the stable nuclei. Figure 6a shows nuclide total activity after 5 years of operation. The total activity of the target is $4.03 \times 10^{17} \mathrm{~Bq}$ and major contributors are ${ }^{187} \mathrm{~W}(31 \%),{ }^{183} \mathrm{~W}_{\mathrm{m}}(24.1 \%),{ }^{185} \mathrm{~W}$ $(15.8 \%),{ }^{181} \mathrm{~W}(3 \%)$ and ${ }^{178} \mathrm{Ta}(1 \%)$. The total activity after 5 years of operation and a shut-down of 9 years is $8.95 \times 10^{14} \mathrm{~Bq}$ (Figure 6b). The list of contributors to the total activity is greatly reduced: ${ }^{3} \mathrm{H}$ is the main one with $76.4 \%$.

Total activity of tungsten target is due to few nuclei next to tungsten target and tritium. As only a few nuclei play a major role, we will proceed nuclei by nuclei to determine uncertainties on residue production yields and activation.

\section{Conclusion}

The INCL4.6-Abla07 benchmark on thin and thick target provides a good agreement (average ratio $\sim 2$ ) with available data even if it is less good as expected with nuclei produced mainly by low energy projectile. When calculation of hazard index will be done, we will be able to validate the contaminant list or define a new one. We still need to assess accuracy of the calculation results and give uncertainties for each contaminant nucleus. Dealing with safety issues, we will take a special care of underestimated isotopes.

\section{References}

[1] A. Boudard, J. Cugnon, S. Leray and C. Volant, Intranuclear cascade model for a comprehensive description of spallation reaction data, Phys. Rev. C 66, 044615 (2002), pp. 1-28.

[2] A.R. Junghans, M. de Jong, H.-G. Clerc, A.V. Ignatyuk, G.A. Kudyaev and K.-H. Schmidt, Projectile-fragment yields as a probe for the collective enhancement in the nuclear level, Nuclear Physics A, 629, (1998), pp. 635-655.

[3] http://monpx.lanl.gov/

[4] http://www-nds.iaea.org/spallation.

[5] J. Cugnon et al., New features of the INCL4 model for spallation reactions, Proc. of the Int. Conf. on Nuclear data for Science and Technology, J. Korean Phys. Soc. 59 (2011), pp. 955-958.

[6] A. Kelić et al., ABLA07 - Towards a complete description of the decay channels of a nuclear system from spontaneous fission to multifragmentation, Proc. of the ICTP-IAEA Advanced Workshop on Model Codes for Spallation Reactions, IAEA INDC(NDS)-0530, Vienna, August 2008, p. 181.

[7] D. Ene, ESS Proposal for the source term definitions nuclides break down lists, private communication (2011).

[8] Yu. E. Titarenko, V. F. Batyaev, A. Yu. Titarenko, M. A. Butko, K. V. Pavlov, S. N. Florya, R. S. Tikhonov, V. M. Zhivun, A. V. Ignatyuk, S. G. Mashnik, S. Leray, A. Boudard, J. Cugnon, D. Mancusi, Y. Yariv, K. Nishihara, N. Matsuda, H. Kumawat, G. Mank and W. Gudowski, Measurement and simulation of the cross sections for nuclides production in ${ }^{\text {nat }} \mathrm{W}$ and ${ }^{181} \mathrm{Ta}$ targets irradiated with $0.04-$ to $2.6-\mathrm{GeV}$ protons, Physics of Atomic Nuclei 74 (2011), pp. 551-572.

[9] R. Michel, M. Gloris, J. Protoschill, U. Herpers, J. Kuhnhenn, F. Sudbrock, P. Malmborg and P. Kubik, Cross section for the production of radionuclides by proton-induced reactions on $\mathrm{W}$, $\mathrm{Ta}, \mathrm{Pb}$ and $\mathrm{Bi}$ from thresholds up to $2.6 \mathrm{GeV}$, Proc. of the Int. Conf. on Nuclear data for Science and Technology, J. of Nuclear Science and Technology, supplement 2 (2002), pp. 242-245.

[10] S. Leray, A. Boudard, J. Cugnon, J.C. David, A. Kelić-Heil, D. Mancusi and M.V. Ricciardi, 
Improved modeling of helium and tritium production for spallation targets, NIM B 268 (2010), pp. 581-586.

[11]J.L. Ullmann, A. Gavron, J. King et al., APT Radionuclide production experiment technical report, Los Alomos National Laboratory Technical report, LA-UR-95-3327 (1995).
[12]Y. Kasugai, T. Kai, F. Maekawa, H. Nakashima, H. Takada, C. Konno, M. Kumajiri, T. Ino and K. Takahashi, Measurement of radioactivity induced by $\mathrm{GeV}$-protons and spallation neutrons using AGS accelerator, Japan Atomic Energy Reserch Institute, JAERI-Research Report 2003-034 (2004). 